\title{
Determination of tetracycline and its major degradation products by liquid chromatography with fluorescence detection
}

\author{
A. Pena ${ }^{\mathrm{a}, *}$, A. Carmona ${ }^{\mathrm{b}}$, A. Barbosa ${ }^{\mathrm{b}}$, C. Lino $^{\mathrm{a}}, \mathrm{I}_{\text {. Silveira }}{ }^{\mathrm{a}}$, B. Castillo $^{\mathrm{b}}$ \\ a Laboratory of Bromatology, Faculty of Pharmacy, University of Coimbra, 3000 Coimbra, Portugal \\ ${ }^{\mathrm{b}}$ Laboratory of Instrumentals Techniques, Department of Analytical Chemistry, Faculty of Pharmacy, Complutense University, \\ Madrid, Spain
}

Received 15 May 1998; received in revised form 4 September 1998; accepted 13 September 1998

\begin{abstract}
A liquid chromatographic method of tetracycline and its major degradation products on a $\mathrm{C}_{8}$-reversed phase column with acidic mobile phase and fluorescence detection is described. The quantification limit, measured as the amount of sample that gave a signal ten times the peak-to-peak noise of the baseline, was: $0.25 \mathrm{ng}$ for tetracycline (TC) and epitetracycline (ETC), $25 \mathrm{ng}$ for and 4-epianhydrotetracycline (EATC) and $50 \mathrm{ng}$ for anhydrotetracycline (ATC) of injected standard. By means of this liquid chromatography (LC) assay TC, ETC, EATC and ATC as main degradation products of tetracycline, can be separated and determined with good sensitivity and specificity within 15 min. (C) 1998 Elsevier Science B.V. All rights reserved.
\end{abstract}

Keywords: Tetracycline; Epitetracycline; Epianydrotetracycline; Anydrotetracycline; Liquid chromatography; Fluorescence detection

\section{Introduction}

Tetracycline antibiotic is widely prescribed in animal husbandry. It is used for the prevention and control of disease and as feed additives to promote weight gain and increase feed conversion efficiency.

Veterinary pharmaceutical formulations of tetracycline (TC) contain small amounts of impurities namely 4-epitetracycline (ETC), anhydro-

\footnotetext{
* Corresponding author. Fax: +351-39-27126.
}

tetracycline (ATC) and 4-epianhydrotetracycline (EATC). Tetracycline used in feed additives are even less pure. The source of the tetracycline used in feeds is the residual tetracycline found in the dried, ground mycelial powder harvested from fermentation tanks [1].

These compounds may also have origin if stored under adverse conditions, e.g. high temperature and humidity [2]. It must be realized that degradation products of tetracyclines can also occur in the stomach [3].

It is important to determine not only the tetracycline and its major degradation products in 
pharmaceutical formulations but also in biological and food samples. Next to a decrease of potency, degradation can lead to toxic degradation products. This is already proven for EATC and ATC $[4,5]$.

Permitted concentrations of these impurities in pharmaceutical formulations are fixed by the European Pharmacopoeia [6].

Since the residues in biological and food samples, are products of metabolism they consist of the parent drug and all compounds derived from it, such as free metabolites. The maximum residue levels (LMR) in foods for TC, established by the European Community (EC) [7] are for the parent compound and its epimer. Therefore, it is also very important to develop methods for its determination in food samples.

The stability of TC is poor under strong acidic and alkaline conditions with reversible formation of epimers at position $\mathrm{C}_{4}$ to 4-epitetracycline (ETC) in weak acid (pH 3) and to anhydro-TC under strong acidic (below $\mathrm{pH}$ 2) conditions [8].

Epimerization on carbon-4 in tetracycline, which is a reversible first-order process, occurs most rapidly between $\mathrm{pH} 3$ and 5 . The presence of a hydroxyl group at $\mathrm{C}_{6}$ favors dehydration and aromatization of the $\mathrm{C}$-ring of tetracycline follow pseudo-first-order kinetics, leading to ATC at very low $\mathrm{pH}$. The epimerization of ATC, and the dehydration of the ETC lead to the formation of EATC [9] (Fig. 1).

The toxic effects of ATC were attributed to the relative position of the dimethylamino group (on $\left.\mathrm{C}_{4}\right)$ [10].

Several papers dealing with the liquid chromatographic determination of tetracyclines and their degradation products have been published $[11,12]$. Those reports deal mainly with the determination of tetracyclines in pharmaceutical preparations, where relatively high concentrations are involved, but it is also important determine their presence in biological and food samples at residual levels. Therefore, it was essential to introduce a more sensitive detection method.

Fluorescence detection of tetracyclines is more specific and also in many cases more sensitive than UV detection normally used [13], and its a important tool in the analysis of tetracycline residues in biological and food samples.

In this paper a LC method with fluorescence detection, according to the Haagsma method [14] validated in our laboratory for the residue analysis of oxytetracycline (OTC), tetracycline (TC) and chlortetracycline (CTC), was tested for the

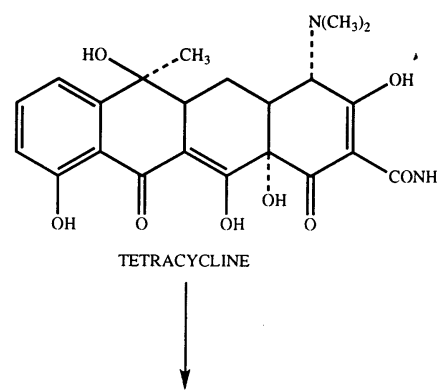

Epimerization
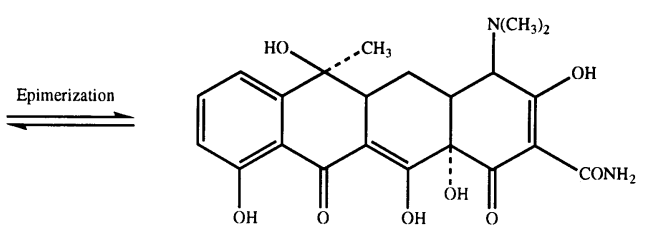

4-EPITETRACYCLINE<smiles>Cc1c2c(c(O)c3c(O)cccc13)C(=O)[C@@]1(O)C(=O)C(C(N)=O)=C(O)[C@H](N(C)C)C1C2</smiles>

$\stackrel{\text { Epimerization }}{\rightleftharpoons}$<smiles>Cc1c2c(c(O)c3c(O)cccc13)C(=O)[C@@]1(O)C(=O)C(C(N)=O)=C(O)C(C)C1C2</smiles>

ANHYDROTETRACYCLINE

4-EPIANHYDROTETRACYCLINE

Fig. 1. Structures of tetracycline and its major related substances. 
analysis of TC, ETC, EATC and ATC. The presented method utilizes the fluorescence produced when tetracyclines reacted with magnesium ions. Most tetracyclines fluorescence in the presence of magnesium ions, and this fluorescence is intensified by the addition of a base such as sodium hydroxide [15].

Tetracycline was found to fluorescence more if heated to produce anhydro-salts before complexation [16]. During dehydration, two double bonds are added to the tetracycline nucleus, producing stable anhydro forms and increasing fluorescence. Therefore, this property can be used for a more sensitive detection of the anhydro forms, measured as anhydro-TC-magnesium complexes.

The objective was develop an LC system which gives fast separation and determination with good sensitivity and selectivity, of TC, ETC, EATC and ATC as main metabolites of tetracycline, suitable for their precise routine analysis in biological and food samples.

\section{Experimental}

\subsection{Instrumentation}

A Gilson model 302, 305 and 307 pumps, a model 7125 loop injector (Rheodyne, Cotati, CA) and a Perkin Elmer LS-3B fluorescence detector operated at an excitation wavelength of $385 \mathrm{~nm}$ and an emission wavelength of $500 \mathrm{~nm}$ were used. The spectral band width was $10 \mathrm{~nm}$ for both excitation and emission. Mobile phase flow $0.8 \mathrm{ml} \mathrm{min}^{-1}$. The derivatization reagent was delivered at a flow rate of $0.45 \mathrm{ml} \mathrm{min}^{-1}$.

The results were recorded on a 3390A chromato- integrator Hewlett Packard. The LC column used was a Chromspher $\mathrm{C}_{8}, 100-3 \mathrm{~mm}$ (Chrompack, Bergen op Zoom, The Netherlands).

\subsection{Reagents}

Caution: tetracyclines are irritants and tetracycline itself is a possible teratogen. Handle tetracyclines standards with care. Prepare mobile phases in well-ventilated chemical fume hood. Avoid contact with skin.

1. Water was purified by distillation and passage through Milli-Q system (Millipore). The water was filtered through a $0.45 \mathrm{~mm}$ filter under vacuum and degassed by ultrasonication.

2. All the solvents were LC grade and were purchased from Carlo Erba (Italy). Oxalic acid, magnesium acetate, boric acid, potassium hydroxide and sodium hydroxide were analytical reagent grade chemical obtained from Merck (Germany).

3. Tetracycline (TC) was obtained from Sigma Chemical (Spain). ETC, EATC and ATC were purchased from European Pharmacopoeia. Individual stock standard solutions of TC, ETC, EATC and ATC were prepared in $100 \mathrm{ml}$ methanol containing $100 \mathrm{mg}$ neat standard into a volumetric flask, and was stored at $-20^{\circ} \mathrm{C}$ in brown glass vials for a maximum period of one month. Tetracycline was corrected to $100 \%$ purity.

The working solutions were a mixture of the four compounds prepared by a serial dilution of the stocks and were stored in brown glass vials at $4^{\circ} \mathrm{C}$. These solutions were prepared immediately before use.

The mobile phases used for analysis containing aqueous oxalic acid solution ( $\mathrm{pH} 2.0 ; 0.01 \mathrm{M}$ ) and $20-40 \%$ of acetonitrile. The mobile phases were filtered through a $0.45 \mathrm{~mm}$ filter under vacuum, degassed by ultrasonication.

The reagent post-column was prepared daily, dissolving $6.0 \mathrm{~g}$ of magnesium acetate, $6.1 \mathrm{~g}$ boric acid and $2.5 \mathrm{~g}$ of potassium hydroxide in $950 \mathrm{ml}$ of water, adjusted with $1 \mathrm{M}$ sodium hydroxide and make up to 11 . Its important to follow the order of addition of these reagents, because precipitations may occur. This solution was filtered through a $0.45 \mu \mathrm{m}$ filter under vacuum and degassed by ultrasonication, was held in a brown glass bottle.

All glassware was cleaned with Extran MA 03, Merck, Germany; $10 \% \mathrm{v} / \mathrm{v}$, rinsed in concentrated acid-dichromate solution, washed thoroughly with tap water, rinsed with deionized water and dried at $80^{\circ} \mathrm{C}$. 
Table 1

Linear regression of the assay response for TC, ETC, EATC and ATC

\begin{tabular}{llllll}
\hline Compound & Slope & Slope SE & Intercept & Intercept SE & Coefficient of correlation \\
\hline ETC & 0.6697 & 0.009429 & 0.0288 & 0.071371 & 0.999 \\
TC & 1.02939 & 0.043006 & 0.0417 & 0.325520 & 0.998 \\
EATC & 0.0746 & 0.003484 & -0.0162 & 0.263743 & 0.996 \\
ATC & 0.1306 & 0.001840 & 0.0993 & 0.139281 & 0.993 \\
\hline
\end{tabular}

\section{Results}

The standard curves were tested for linearity in the range of $2.5-25 \mathrm{ng}$ for ETC and TC, and 50-250 ng for EATC and ATC, of injected amount.

The linear regressions of the assay response for TC, ETC, EATC and ATC are shown in Table 1. The correlation coefficients for the regression equation for TC, ETC, EATC and ATC were greater than 0.993 , showing the standard curve to be linear within the range of standards used.

The quantification limits, measured as that amount of sample that gave a signal ten times the peak-to-peak noise of the baseline, were: $0.25 \mathrm{ng}$ for TC and ETC, $25 \mathrm{ng}$ for EATC and $50 \mathrm{ng}$ for ATC of injected standard.

The quantification of small amounts of impurities can be realized owing to the good separation and sensitivity.

Within-day precision were determined by analysing five standard solutions at two levels of concentration (ETC and TC at 25 and $2.5 \mathrm{ng}$ and EATC and ATC at 250 and $50 \mathrm{ng}$ ), whilst between-day precision were determined by analysing the same standard solutions on 5 successive days. We obtained coefficients of variation between 2.4 and $3.5 \%$ and 9.0 and $4.1 \%$ respectively, showing a good precision of the method.

The individual stock standard solutions of TC, ETC, EATC and ATC were prepared in methanol and stored at $-20^{\circ} \mathrm{C}$ in brown glass vials. We did not observe significant alterations over a maximum period of 1 month. The working solutions were a mixture of the four compounds prepared by a serial dilutions of the stocks with methanol, in brown glass vials, and were prepared daily, immediately before use. Some care was taken with these solutions: they were always kept at $4^{\circ} \mathrm{C}$ and protected from light, immediately after the injection in the chromatographic system, they were not allowed to stand in the laboratory at room temperature. Under these conditions no appreciable decomposition was observed in these solutions for approximately 1 working day $(8-12 \mathrm{~h})$.

The good repeatability mentioned in the calibration is an indication for the good stability of this compounds during the chromatographic analysis.

\section{Discussion}

The quantification limits achieved by the present method are in agreement with the MRL established by the European Comminity for TC and its epimer in foods and in the same chromatographic run we can also averiguate the presence of the toxic EATC and ATC. Since the anhydroTC-magnesium complexes are highly fluorescent the method is more sensitive for these compounds permitting the separation of very small amounts of EATC and ATC and small amounts of ETC can be separated from TC, according to the European Pharmacopoeia permitted concentrations for pharmaceutical preparations.

Chromatography of anhydrotetracyclines is some what more difficult to achieve than that the parent compounds because of the lower polarity of the former.

A study on reversed-phase LC of TC and its degradation products using acid mobile phases was first published by Knox et al. [17].

The method presented utilizes a $\mathrm{C}_{8}$ Chromospher column at room temperature with acetonitrile- $-0.01 \mathrm{M}$ oxalate buffer $(\mathrm{pH}$ ) as the mobile 
phase. Tetracyclines form chelate complexes with ions at $\beta$-diketones $\left(\mathrm{C}_{10}-\mathrm{C}_{12}\right)$ and carboxyamide $\left(C_{2}\right)$ [18] and bind with silanol groups in the stationary phase [19]. In an acidic medium $(\mathrm{pH}$ $1-2.5)$ the tetracycline molecule is fully protonated and exists in its cationic form [20,21] and can be paired with a suitable anion such as oxalate [22]. On the other hand, all tetracyclines had the best asymmetric values at $\mathrm{pH} 2$ [23].

Following elution, tetracyclines are derivatized with $\mathrm{Mg}^{2+}$ ions at room temperature to produce a highly fluorescent derivative. The fluorescence detector is set with an excitation wavelength of $385 \mathrm{~nm}$ and an emission wavelength of $500 \mathrm{~nm}$.

The development of the fluorimetric method was based upon experience obtained with the analysis of OTC, TC and CTC. The fluorescence response is dependent upon $\mathrm{pH}$. For a maximum fluorescence a $\mathrm{pH}$ greater than 8 is essential, and is associated with the ionized form of the phenolic- $\beta$-diketone site of these molecules. The $\mathrm{pH}$ is adjusted with the addition of the post-column reagent, prepared in alkaline solution at $\mathrm{pH} 9$.

The fluorescence response is also dependent upon the flow rate of derivatization reagent. In our study the fluorescence response reached a maximum at $0.45 \mathrm{ml} \mathrm{min}^{-1}$. Post-column derivatization does not directly affect the chromatographic properties of the tetracyclines, however the reaction chemistry must be rapid on the chromatographic time scale in order to preserve the chromatographic information.

Post-column derivatization has also the advantage that a separate sample treatment step is not required and the analytes are better separated from interferences prior to derivatization.

Preliminar work was carried out on isocratic analysis, making several variations of the ratio of organic modifier and aqueous oxalic acid in the mobile phase.

The composition of acid mobile phases must be established very well, because complete separation of TC and ETC is obtained only with mobile phases which are too weak to eluted the more strongly retained EATC and ATC within a reasonable time. The retention decrease directly with concentration of acetonitrile in the mobile phase. We can obtain shorter retention times of EATC and ATC altering the concentration of organic modifier.

We have tried several modifications of the percentage of acetonitrile in the mobile phase, so those anhydrotetracyclines eluted after the tetracycline with good resolution, permitting also the separation of tetracycline and his epimer.

The isocratic analysis using acetonitrile-oxalic acid solution $(\mathrm{pH} 2.0 ; 0.01 \mathrm{M})(30: 70, \mathrm{v} / \mathrm{v})$ as mobile phase, allows the separation of the four compounds but with bad resolution.

With $20 \%$ of acetonitrile the resolution between ETC and TC was good but EATC and ATC eluted very late with bad resolution.

An increase of the acetonitrile concentration to $40 \%$ finally enable the elution of EATC and ATC but with this mobile phase ETC overlapped with the TC peak.

Several variations of the ratio of acetonitrile in the acid mobile phases did not yield substantially better separations, and some improvement was achieved by the use of gradient elution as we can observe in Fig. 2, showing greater resolution of these compounds. The gradient was chosen based in this work experience, to allow for optimum separation. We have applied the follow gradient: from 0 to $5 \mathrm{~min} 20 \%$ of acetonitrile and $80 \%$ of oxalic acid ( $\mathrm{pH} 2.0 ; 0.01 \mathrm{M}$ ), from 5 to $16 \mathrm{~min}$ $40 \%$ of acetonitrile and $60 \%$ of oxalic acid $(\mathrm{pH}$ $2.0 ; 0.01 \mathrm{M}$ ) and at $17 \mathrm{~min} 30 \%$ of acetonitrile and $70 \%$ of oxalic acid $(\mathrm{pH} 2.0 ; 0.01 \mathrm{M})$, at a

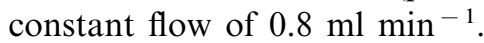

\section{Conclusions}

Methanol was chosen as the universal solvent for the tetracyclines, because of its ability to dissolve the tetracyclines and its miscibility with aqueous and organic solvents. Methanol was also chosen because aqueous solvents tend to accelerate degradation of tetracyclines compounds.

Because many reversed-phase materials are unstable at $\mathrm{pH}$ lower than 2-3 it was necessary to flush the columns with a neutral solvent (e.g. water-acetonitrile 50:50) for $1 \mathrm{~h}$ at the end of each working day [22]. This practice contributed markedly to the prolonging of the column life. 
Although the $\mathrm{C}_{18}$-bound materials are more suitable for analysing the impurities of TC, showing better resolution $\left(R_{\mathrm{s}}\right)$ values, we obtained good symmetrical peaks using a Chromspher $\mathrm{C}_{8}$ column. Our results show that even though a $\mathrm{C}_{8}$ column was used, we obtained good performance. This method enables good separations of TC, ETC, EATC and ATC.

The methodology reported herein use a simple solvent system containing a low concentration of buffer, avoiding the drawbacks related with its use for the chromatographic system. Its also allows analysis of the tetracyclines and its degradation products with the same chromatographic column.

Although the analysis was performed at $\mathrm{pH} \mathrm{2,}$ this system was proven not to cause any epimerization of TC. Formation of EATC and ATC due to partial degradation of ETC and TC in the strong acidic mobile phase also was not observed.

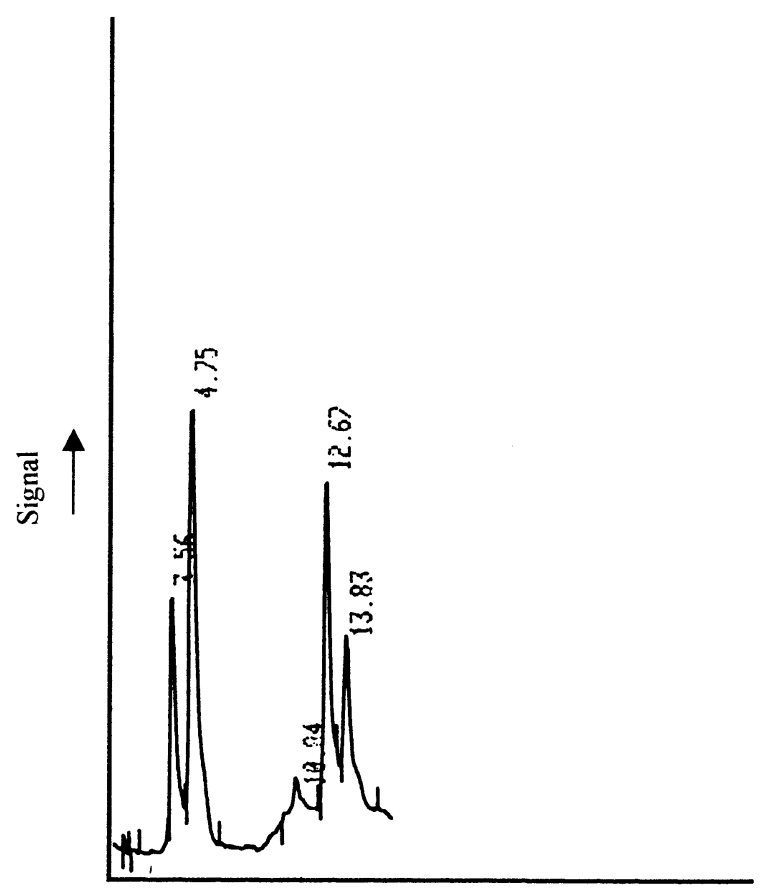

Retention time

Fig. 2. Chromatogram in gradient analysis indicated. ETC-Tr 3.56; TC-Tr 4.75; EATC-Tr 12.67 and ATC-Tr 13.83.
The epimer of TC is always eluted first, while its anhydro-forms are most retained in agreement with the polarity of the tetracycline compounds. Accordingly to polarity of the chromatographic column anhydro-forms, EATC and ATC, are eluted with good resolution only with a high percentage of acetonitrile in the mobile phase.

With this LC method we can differentiate between the tetracycline and their major degradation products, at residue level within $15 \mathrm{~min}$. Thus it is possible to ascertain whether the tetracyclines determined in biological and food samples are the intact molecule originally applied or some fluorescent degradation and food products. The high degree of selectivity achieved in using a postcolumn reaction and fluorescence detection, that is less prone to interference from other compounds in the sample matrix, has considerable potential as a basis for the development of a method for the determination of these compounds in biological and food samples, since we will can minimize the complex time consuming sample extraction and clean-up procedures.

\section{References}

[1] R.B. Ashworth, J. Assoc. Anal. Chem. 68 (1985) 10131018.

[2] R.F. Lindawer, D.M. Cohen, K.P. Munnely, Anal. Chem. 48 (1976) 1731-1734.

[3] H.J.E.M. Reewijk, V.R. Tjaden, J. Chromatogr. 353 (1986) 339-350.

[4] H. Oka, M. Suzuki, J. Chromatogr. 314 (1984) 303-311.

[5] A. Azalos, Chromatographia 10 (1985) 313-323.

[6] European Pharmacopeia, 3rd edn., 1997.

[7] Commission Regulation no. 281/96, Off. J. Eur. Commun., L37/9-L37/11, 14 February, 1996.

[8] H. Oka, H. Nakagawa, K.-I. Harada, J.D. Mac Neil, in: AOAC Int. (Eds.), Chemical Analysis for Antibiotic used in Agriculture, AOAC Int., 1995, pp. 332-346.

[9] N.H. Khan, P. Wera, E. Roets, J. Hoogmartens, J. Liq. Chromatogr. 13 (1990) 1351-1374.

[10] H.F dos Santos, W.B.de Almeida, M.C. Zerner, J. Pharm. Sci. 87 (1998) 190-195.

[11] N.H. Khan, P. Wera, J. Hoogmartens, J. Liq. Chromatogr. 13 (1990) 1351-1374.

[12] C. Hendrix, E. Roets, J. Crommen, J de Beer, E. Porqueras, W. Van der Bossche, J. Hoogmartens, J. Liq. Chromatogr. 16 (1993) 3321-3329.

[13] J.H. Knox, J. Jurand, J. Chromatogr. 110 (1975) $103-$ 115. 
[14] R.J. McCracken, W.J. Blanchflower, S.A. Haggan, D.G. Kennedy, Analyst 120 (1995) 1763-1766.

[15] N. Haagsma, P. Scherpenisse, in: N. Haagsma, A. Ruiter, P.B. Czedik-Eysenberg (Eds.), Proceedings of the Euroresidue II Conference Veldohoven, 1993, pp. 342-346.

[16] H. Poiger, Ch. Schlatter, Analyst 101 (1976) 808-814.

[17] D. Hall, J. Pharm. Pharmacol. 28 (1976) 420-422.

[18] Y.Y. Lee, W. Evrett, J. Am. Chem. Soc. 103 (1981) 5221.
[19] C. Bogert, A.M. Kroon, J. Pharm. Sci. 70 (1981) 186189.

[20] C.R. Stephens, K. Murai, K. Brunings, R.B Woodward, J. Am. Chem. Soc. 78 (1956) 4155-4158.

[21] J.H. Knox, J. Jurand, J. Chromatogr. 186 (1979) 763-782.

[22] F. Kramer-Hraczynska, J. Chromatogr. Sci. 29 (1991) 107-113.

[23] H. Oka, K. Uno, K.-I. Harada, K. Yasada, M. Suzuki, J. Chromatogr. 298 (1984) 435-443. 